\title{
On the Minimum Number of Wavelengths in Multicast Trees in WDM Networks
}

\author{
Yingyu Wan, Weifa Liang \\ Department of Computer Science, The Australian National University, Canberra, ACT 0200, Australia
}

\begin{abstract}
We consider the problem of minimizing the number of wavelengths needed to connect a given multicast set in a multihop WDM optical network. This problem was introduced and studied by Li et al. (Networks, 35(4), 260-265, 2000) who showed that it is NP-complete. They also presented an approximation algorithm for which they claimed an approximation ratio of $c(1+2 \log \Delta)$, where $c$ is the maximum number of connected components in the subgraph induced by any wavelength and $\Delta$ is the maximum number of nodes in any connected component induced by any wavelength. In this article we present an example demonstrating that their claim cannot be correct-the approximation ratio is $\Omega(n)$, even though the subgraph induced by every wavelength is connected, where $n$ is the number of nodes in the network. In fact, we show that the problem cannot be approximated within $O\left(2^{\log ^{1 / 2-\varepsilon} m}\right)$ unless NP $\subseteq$ $D T I M E\left(n^{\text {poly log } n}\right)$ for any constant $\varepsilon>0$, where $m$ is the number of edges in the network. We complement this hardness result by presenting a polynomial-time algorithm with an approximation ratio of $(1+\ln 3+2 \log \Delta)$ when the subgraph induced by every wavelength is connected, and an approximation ratio of $O(\sqrt{(n \log \Delta) / o p t)}$ in the general case, where opt is the number of wavelengths used in an optimal solution and $1 \leq$ opt $\leq n-1$. () 2004 Wiley Periodicals, Inc. NETWORKS, Vol. 45(1), 42-48 2005
\end{abstract}

Keywords: multicast; wavelength assignment and routing; the minimum number of wavelengths; WDM networks

\section{INTRODUCTION}

The emerging optical network offers the possibility of interconnecting hundreds to thousands of users, covering a local to wide area, and providing capacities substantially exceeding conventional electronic networks. The network promises data transmission rates several orders of magnitude higher than current electronic networks. The key to high speed in the network is to maintain the signal in optical form rather than conventional electronic form. The high

Received July 2003; accepted November 2004.

Correspondence to: W. Liang; e-mail: wliang@cs.anu.edu.au

Contract grant sponsor: Australian Research Council (Discovery Schemes); contract grant numbers: DP0342900 and DP0449431 DOI 10.1002/net.20048

Published online in Wiley InterScience (www.interscience.wiley.com). (C) 2004 Wiley Periodicals, Inc. bandwidth of fiber-optic links is utilized through Wavelength-Division Multiplexing (WDM) technology, which supports the propagation of multiple laser beams through a single fiber-optic link provided that each laser beam uses a distinct optical wavelength.

Multicast communication is a one-to-many or many-tomany communication. A multicast connection request typically involves the transport of information between a single sender (source) and multiple receivers (destinations). Multicast applications include video conferencing, entertainment distribution, tele-classrooms, distributed data processing, etc. $[1,12,14]$. With growing demand for these services and the availability of high bandwidth, future networks such as WDM networks must be equipped to handle multicast communication in an efficient manner, and the most popular solutions to multicast routing involve tree constructions. In a WDM optical network, establishing a multicast connection involves routing and wavelength assignment, where routing is to establish a multicast tree rooted at the source and spanning all destination nodes, while the wavelength assignment is to assign wavelengths to the edges in the routing tree.

There are two types of architectures for WDM optical networks. One is the single-hop network [10], in which a multicast connection request is assigned a single wavelength only because no wavelength conversion at relay nodes is available. It is desirable to transmit a message from the source to all destinations on a single wavelength, but is not generally feasible to do so, because a specific wavelength on some links is being used by other routing traffic, and is not available for a new multicast connection request. Also, there is the physical constraint that each optical link can only carry a limited number of wavelengths. Instead, the multihop WDM optical network has been introduced [11], in which each node is equipped with a set of fixed transmitters and receivers that can convert an incoming wavelength to another outgoing wavelength. Thus, to respond to a multicast connection request, a routing tree is established for it with different wavelengths assigned to the tree edges.

Multicast routing in WDM networks is an active research topic. There are several important optimization objectives for it, which include minimizing the total cost of the net- 
work resources, minimizing the number of wavelength conversion, and minimizing the number of wavelengths used. In this article we deal with the multicast problem in a multihop WDM optical network with an optimization objective to find a multicast tree such that the number of wavelengths used in the tree is minimized.

\subsection{Related Work}

Let $D$ be the multicast set, that is, the set of nodes involved in a multicast connection request. If the optimization objective for a multicast connection request is the total cost of the network resources used, several studies have been carried out $[2,3,7,8]$. For example, when $|D|=1$, the problem becomes the optimal semilightpath problem [3], for which Chlamtac et al. [3] presented an algorithm, which takes $O\left(k^{2} n+k n^{2}\right)$ time, where $k$ is the number of wavelengths and $n$ is the number of nodes in the network. Liang and Shen [7] later provided an improved algorithm for the problem. Their algorithm takes $O\left(k^{2} n+k m+k n \log (k n)\right)$ time, and can be implemented in the distributed environment, where $m$ is the number of edges in the network. When $|D|>1$, Liang and Shen [8] proposed an approximation algorithm for it. Chen and Wang [2] recently provided an exact solution for the problem in a very special WDM optical network-the tree network. Li et al. [6] considered the multicast problem with minimizing the number of wavelengths in the multicast tree as their optimization objective (which we refer to as the minimum wavelength multicast problem). They showed that the problem is NP-complete, and provided an approximation algorithm with the claimed approximation ratio of $(1+2 \log \Delta)$ if the induced subgraph by any wavelength is connected; otherwise $c(1+2$ $\log \Delta$ ), where $c$ is the maximum number of connected components in the subgraph induced by any wavelength and $\Delta$ is the maximum number of nodes in any connected component induced by any wavelength. However, their algorithm cannot deliver a solution with the promised approximation ratio, which will be shown by a counterexample in the appendix of this article. Motivated by their work, in this article we will provide an approximation algorithm for the problem with a guaranteed approximation ratio. In addition, there have been several other studies for constructing multicast trees in WDM optical networks with other optimization objectives. For example, Zhang et al. [15] considered the multicast problem by focusing on the limited splitting power of optical switches, and provided heuristic algorithms for the problem. Znati et al. [16] dealt with the problem by decoupling the delay from the other cost of network resources, and presented several heuristic algorithms for finding a multicast tree satisfying both delay and cost optimization objectives. Jia et al. [5] considered the routing congestion issue in a single hop (all-optical) network by proposing two heuristic algorithms for a multicast problem that aims to minimize the total cost of a multicast tree and to bound the maximum delay between the source and the destinations. Libeskind-Hadas and Melhem [9] in- vestigated the multicast communication in circuit-switched multihop networks by showing it is polynomially solvable when the optimization objective is the wavelength assignment only, despite the fact that the general multicast problem is NP-Hard.

\subsection{Our Contributions}

In this article our major contributions for the concerned problem are as follows. We first show that the minimum wavelength multicast problem cannot be approximated within $O\left(2^{\log ^{1 / 2-\varepsilon} m}\right)$ unless $N P \subseteq D T I M E\left(n^{\text {poly } \log n}\right)$ for any constant $\varepsilon>0$. We then present an approximation algorithm with an approximation ratio of $O(\sqrt{ } n \log \Delta) /$ $o p t$ ) for the problem, where opt is the number of wavelength in an optimal solution.

\subsection{Paper Organization}

The rest of the article is organized as follows. In Section 2 we introduce notations and the problem definition. In Section 3 we show the approximation complexity of the minimum wavelength multicast problem. In Section 4 we propose an approximation algorithm for the problem, and in Section 5 we conclude the article.

\section{PRELIMINARIES}

The multihop WDM optical network can be modeled by an undirected graph $G=(V, E, \Lambda)$, where $V$ is a set of nodes (vertices), $E$ is a set of links (edges), and $\Lambda$ is a set of wavelengths in $G$. Let $n=|V|, m=|E|$, and $\Lambda=\left\{\lambda_{1}\right.$, $\left.\lambda_{2}, \ldots, \lambda_{|\Lambda|}\right\}$. Associated with each node $v \in V$, there is a fully convertible wavelength switch that can convert any incoming wavelength to any outgoing wavelength if necessary. Associated with each link $e \in E$, there is a set $\Lambda(e)(\subseteq$ $\Lambda)$ of wavelengths available on it. Unless otherwise specified, we use term edge and link interchangeably. For each wavelength $\lambda$, let $G_{\lambda}=\left(V_{\lambda}, E_{\lambda}\right)$ be the induced subgraph of $G(V, E, \Lambda)$, where $E_{\lambda}=\{e \in E \mid \lambda \in \Lambda(e)\}$ and $V_{\lambda}$ $=\{v \in V \mid \exists e=(u, v) \in E, \lambda \in \Lambda(e)\}$. Each node in $V_{\lambda}$ is covered by $\lambda$, and each edge in $E_{\lambda}$ is covered by $\lambda$ also.

Given a multicast connection request set $D \subseteq V$, the minimum wavelength multicast problem (MWM for short) is to construct a tree $T=\left(V_{T}, E_{T}\right)$ with a wavelength assignment function $l_{T}: E_{T} \rightarrow \Lambda$ such that the number of wavelengths used in $T$ is minimized. The tree and the wavelength assignment function satisfy that $D \subseteq V_{T} \subseteq V$, $E_{T} \subseteq E$ and $l_{T}(e) \in \Lambda(e)$ for every edge $e \in E_{T}$. In other words, the objective of MWM is to find such a multicast tree $T$ with $\min _{T}\left|\left\{l_{T}(e) \mid e \in E_{T}\right\}\right|$.

\section{APPROXIMATION COMPLEXITY OF MWM}

We prove the approximation complexity of MWM by a reduction from the symmetric label cover problem (SLC). 


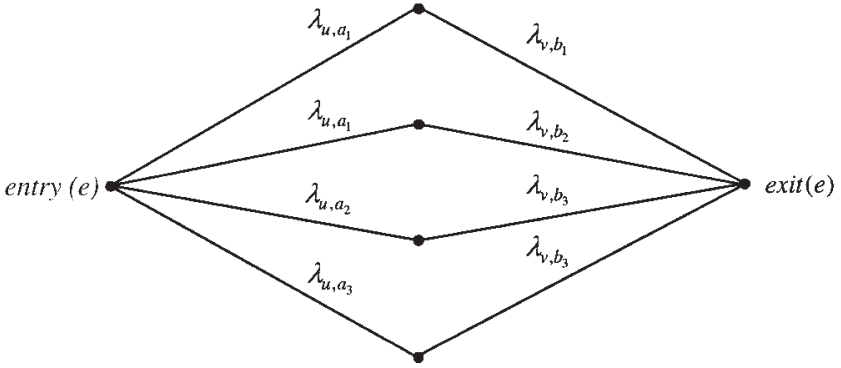

FIG. 1. An edge gadget.

The input of SLC is a bipartite graph $H=(L, R, E)$ and two sets of labels: $A$ and $B . L$ and $R$ are the disjoint sets of nodes, and $E \subseteq L \times R$. $A$ is used on $L$ only, and $B$ on $R$ only. For each edge $e \in E$ there is an admissible set $Q_{e} \subseteq$ $A \times B$. A feasible solution is a label assignment $A_{u} \subseteq A$ for each node $u \in L$ and $B_{v} \subseteq B$ for each node $v \in R$ such that there is at least $(a, b) \in Q_{e}$ with $a \in A_{u}$ and $b \in B_{v}$ for every edge $e=(u, v)$. The objective is to minimize $\sum_{u \in L}\left|A_{u}\right|+\sum_{v \in R}\left|B_{v}\right|$. Let $q=\sum_{e \in E}\left|Q_{e}\right|$. Note that the size of an SLC instance is equal to $\Theta(q)$. Dodis and Khanna [4] proved that the existence of a polynomial time $O\left(2^{\log ^{1 / 2-\varepsilon_{q}}}\right)$-approximation algorithm for SLC, for any constant $\varepsilon>0$, implies that every problem in $N P$ can be solved

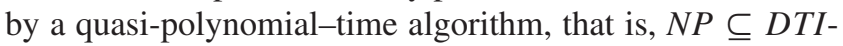
$M E\left(n^{\text {poly } \log n}\right)$.

In the following, we reduce SLC to a special case of MWM, where $|D|=2$, which is referred to as the minimum wavelength unicast problem (MWU for short). The objective of MWU is to find a routing path between the two nodes with the minimum number of wavelengths in the path.

Theorem 1. $M W U$ cannot be approximated within $O\left(2^{\log ^{1 / 2-\varepsilon_{m}}}\right)$ for any constant $\varepsilon>0$, unless that $N P \subseteq$ DTIME $\left(n^{\text {poly } \log n}\right)$.

Proof. Given an SLC instance $H=(L, R, E)$, let $A$ $=\left\{a_{1}, a_{2}, \ldots, a_{|A|}\right\}$ and $B=\left\{b_{1}, b_{2}, \ldots, b_{|B|}\right\}$. For each edge $e=(u, v) \in E$, an edge gadget is constructed, which is shown in Figure 1. The gadget consists of several parallel paths between the entry node entry $(e)$ and the exit node exit( $e$ ), and each parallel path consists of two edges. For each $(a, b) \in Q_{e}$, there is a corresponding path in the gadget, which is referred to as a track of $(a, b)$. On the track of $(a, b)$, the edge adjacent to entry $(e)$ is assigned wavelength $\lambda_{u, a}$ and the edge adjacent to exit(e) wavelength $\lambda_{v, b}$. An example with $Q_{e}=\left\{\left(a_{1}, b_{1}\right),\left(a_{1}, b_{2}\right),\left(a_{2}, b_{3}\right)\right.$, $\left.\left(a_{3}, b_{3}\right)\right\}$ is shown in Figure 1 .

Let $e_{1}, e_{2}, \ldots, e_{|E|}$ be the sequence of edges in $H$. Concatenate the edge gadgets of the edges in the sequence together, that is, the exit node exit $\left(e_{i}\right)$ and the entry node entry $\left(e_{i+1}\right)$ are the same node after concatenation for all $i$, $i=1, \ldots,|E|-1$. Denote by $G$ the resulting graph. Let $D=\left\{\operatorname{entry}\left(e_{1}\right), \operatorname{exit}\left(e_{i+1}\right)\right\}$. Then, an instance of MWU is obtained.
Now, consider a path $P=\left(V_{P}, E_{P}\right)$ in $G$ between nodes entry $\left(e_{1}\right)$ and exit $\left(e_{k}\right)$, where $V_{P}$ is the set of nodes and $E_{P}$ is the set of edges in $P$. For each edge $e \in E_{P}$, let $l_{P}(e)$ be the wavelength assigned to $e$. A label assignment for $H$ is constructed as follows.

$$
\begin{aligned}
& A_{u}=\left\{a \in A \mid \exists e \in E_{P}, l_{P}(e)=\lambda_{u, a}\right\} \quad \text { for each } u \in L, \\
& B_{v}=\left\{b \in B \mid \exists e \in E_{P}, l_{P}(e)=\lambda_{v, b}\right\} \quad \text { for each } v \in R .
\end{aligned}
$$

For each edge $e=(u, v)$ in $H, P$ contains at least one track in the edge gadget that corresponds to $e$. Let $\lambda_{u, a}$ and $\lambda_{v, b}$ be the two wavelengths assigned to the two edges in the track, respectively. Then, $a \in A_{u}$ and $b \in B_{v}$. Thus, the resulting label assignment is a feasible solution of the instance of SLC, and

$$
\begin{array}{r}
\sum_{u \in L}\left|A_{u}\right|+\sum_{v \in R}\left|B_{v}\right|=\sum_{u \in L}\left|\left\{a \in A \mid \exists e \in E_{P}, l_{P}(e)=\lambda_{u, a}\right\}\right| \\
+\sum_{v \in R}\left|\left\{b \in B \mid \exists e \in E_{P}, l_{P}(e)=\lambda_{v, b}\right\}\right| \\
=\left|\left\{l_{P}(e) \mid e \in E_{P}\right\}\right| .
\end{array}
$$

On the other hand, given a feasible label assignment $A_{u}$ and $B_{v}$ for $H$, a path $P$ in $G$ between nodes entry $\left(e_{1}\right)$ and exit $\left(e_{|E|}\right)$ can be constructed as follows. For each edge ( $u$, $v) \in E$, we know that there is a label $(a, b) \in A_{u} \times B_{v}$ satisfying $(a, b) \in Q_{(u, v)}$. Add the track of $(a, b)$ in the edge gadget corresponding to $(u, v)$ into $P$, and assign the two edges in the track with wavelengths $\lambda_{u, a}$ and $\lambda_{v, b}$, respectively. As a result, there is a simple path between entry $\left(e_{1}\right)$ and exit $\left(e_{|E|}\right)$, and the total number of wavelengths used in path $P$ is no more than $\Sigma_{u \in L}\left|A_{u}\right|+\sum_{v \in R}$ $\left|B_{v}\right|$.

The above polynomial reduction actually establishes a cost-preserving reduction between a feasible solution of SLC and a feasible solution of MWU. Note that in the above reduction, the number of edges in the MWU instance defined is $\Theta\left(\sum_{e \in E}\left|Q_{e}\right|\right)$. Thus, the theorem follows, by the result of [4].

Corollary 1. MWM cannot be approximated within $O\left(2^{\log ^{1 / 2-\varepsilon_{m}}}\right)$ for any constant $\varepsilon>0$, unless that $N P \subseteq$ DTIME $\left(n^{\text {poly } \log (n)}\right)$.

\section{APPROXIMATION ALGORITHM FOR MWM}

In this section we aim to develop a nontrivial approximation algorithm for MWM, which delivers a solution within an approximation ratio of $O(\sqrt{(n \log \Delta) / o p t})$. We start with the following two assumptions. $(A)$ For any wavelength $\lambda$, the induced subgraph $G_{\lambda}=\left(V_{\lambda}, E_{\lambda}\right)$ of $G$ is connected. (B) For any wavelength $\lambda,\left|V_{\lambda} \cap D\right| \leq 1$, that is, $\lambda$ covers at most one node in $D$.

For the convenience of discussion, in the following we 
first assume that assumptions $A$ and $B$ hold. We then show how to remove these assumptions.

\subsection{Algorithm with Assumptions A and B}

Let the network $G(V, E, \Lambda)$ satisfy assumptions $A$ and $B$. We construct an auxiliary graph $G_{A B}=\left(V_{A B}, E_{A B}\right)$ and reduce MWM on $G$ to the minimum Steiner tree problem on $G_{A B}$. The construction of $G_{A B}$ is as follows. For each wavelength $\lambda \in \Lambda$, there is a wavelength node $v_{\lambda}$ in $V_{A B}$. For each node $u \in D$, there is a terminal node $t_{u}$ in $V_{A B}$. Denote by $V_{\Lambda}$ the set of wavelength nodes and $V_{D}$ the set of terminal nodes. $V_{A B}=V_{\Lambda} \cup V_{D}$. For any two wavelengths $\lambda_{i}, \lambda_{j} \in \Lambda$, if $V_{\lambda_{i}} \cap V_{\lambda_{j}} \neq \varnothing$, that is, the two wavelengths cover at least one common node in $G$, add an edge $\left(v_{\lambda_{i}}, v_{\lambda_{j}}\right)$ between the corresponding wavelength nodes $v_{\lambda_{i}}$ and $v_{\lambda_{j}}$. Denote by $E_{\Lambda}$ the set of all such edges. For each node $u$ $\in D$, if $u$ is covered by a wavelength $\lambda_{i}$ in $G$, add an edge $\left(t_{u}, v_{\lambda_{i}}\right)$ between the terminal node $t_{u}$ and wavelength node $v_{\lambda_{i}}$. Denote by $E_{D}$ the set of all such edges. Then $E_{A B}=E_{\Lambda}$ $\cup E_{D}$. Assign each edge in $E_{A B}$ with weight 1 .

Given a multicast tree $T$ for MWM in $G$, it can be converted to a Steiner tree $T_{A B}$ in $G_{A B}$ spanning the nodes in $V_{D}$. For any two nodes $u_{i}, u_{j} \in D$, there is a path in $T$ between $u_{i}$ and $u_{j}$. Without loss of generality, let the wavelengths assigned to the edges along the path from node $u_{i}$ to node $u_{j}$ be in the order $\lambda_{1}, \lambda_{2}, \ldots, \lambda_{k}$. Then, $u_{i}$ is covered by $\lambda_{1}$, and there is an edge $\left(t_{u_{i}}, v_{\lambda_{1}}\right)$ in $G_{A B}$. Similarly, there is an edge $\left(t_{u_{j}}, v_{\lambda_{k}}\right)$ in $G_{A B}$. For any two wavelengths $\lambda_{t}$ and $\lambda_{t+1}(1 \leq l<k)$, the corresponding wavelength nodes $v_{\lambda_{t}}$ and $v_{\lambda_{t+1}}$ must be adjacent in $G_{A B}$ because $\lambda_{t}$ and $\lambda_{t+1}$ cover at least a common node in $G$. So, there is an edge $\left(v_{\lambda_{t}}\right.$, $v_{\lambda_{t+1}}$ ) in $G_{A B}$. The subgraph of $G_{A B}$ induced by the node set $\left\{t_{u_{i}}, t_{u_{j}}\right\} \cup\left\{v_{\lambda_{1}}, \ldots, v_{\lambda_{k}}\right\}$ is thus connected. Let $\Lambda_{T}$ be the set of wavelengths used in $T$ and $V_{\Lambda_{T}}$ the set of wavelength nodes in $G_{A B}$ corresponding to the wavelengths of $\Lambda_{T}$. The subgraph of $G_{A B}$ induced by the node set $V_{D} \cup V_{\Lambda_{T}}$ is connected, too, by the similar argument. The Steiner tree $T_{A B}$ can then be easily obtained from this subgraph by the removal of some edges.

On the other hand, given a Steiner tree $T_{A B}$ in $G_{A B}$ spanning the nodes in $V_{D}$, it can be transformed into a multicast tree $T$ for MWM in $G$. For any two nodes $t_{u_{i}}, t_{u_{\text {i }}}$ $\in V_{D}$, there is a path in $T_{A B}$ between them. Without loss of generality, let $t_{u_{i}}, v_{\lambda_{1}}, \ldots, v_{\lambda_{k}}, t_{u_{j}}$ be the sequence of nodes in the path. Following assumption $A$, the subgraph of $G$ induced by the edge set $\cup_{1 \leq l \leq k} E_{\lambda_{l}}$ is connected and the two nodes $u_{i}$ and $u_{j}$ are included in the subgraph. Let $\Lambda_{T_{A B}}$ be the set of wavelengths corresponding to the wavelength nodes in $T_{A B}$. Then, the subgraph of $G$ induced by the edge set $\cup_{\lambda \in \Lambda_{A B}} E_{\lambda}$ is connected, and all the nodes in $D$ are included in the subgraph. The multicast tree $T$ can be obtained from the subgraph easily. Let $w$ be the number of wavelengths used in the multicast tree $T$ constructed from $T_{A B}$.

Let $w_{O P T}^{G}$ be the number of wavelengths in an optimal multicast tree in $G$ and $w_{O P T}^{G_{A B}}$ be the number of edges in a minimum Steiner tree in $G_{A B}$. Let $w_{A B}$ be the number of edges in the Steiner tree $T_{A B}$ in $G_{A B}$.

Lemma 1. (i) $w \leq w_{A B}+1-|D|$; (ii) $w_{O P T}^{G}=w_{O P T}^{G_{A B}}+$ $1-|D| ;($ iii $) w_{O P T}^{G} \geq|D|$; and (iv) $w_{O P T}^{G_{A B}} \geq 2|D|-1$.

Proof. Following the conversion from $T_{A B}$ to $T$, it is easy to see that $w$ is no more than the number of wavelength nodes in $T_{A B}$, which is $w_{A B}+1-|D|$.

From the above argument, we know $w_{O P T}^{G} \leq w_{O P T}^{G_{A B}}+1$ - $|D|$. On the other hand, $w_{O P T}^{G} \geq w_{O P T}^{G}+1-|D|$, following the conversion from $T$ to $T_{A B}$. Thus, $w_{O P T}^{G}=$ $w_{O P T}^{G_{A B}}+1-|D|$.

It is easy to show that $w_{O P T}^{G} \geq|D|$, because each wavelength covers at most one node in $D$ by assumption $B$. At the same time, $w_{O P T}^{G_{A B}} \geq 2|D|-1$ due to the fact that $w_{O P T}^{G}=w_{O P T}^{G_{A B}}+1-|D|$ and $w_{O P T}^{G} \geq|D|$.

We are ready to present the following algorithm.

Algorithm MWM-AB( $G, D)$

Input: $G(V, E, \Lambda)$ satisfying assumption $A$ and $B, D \subseteq V$ Output: $T$ spanning the nodes in $D$ with wavelength assignment function $l_{T}$

Begin

1. Construct $G_{A B}=\left(V_{\Lambda} \cup V_{D}, E_{\Lambda} \cup E_{D}\right)$ from $G$.

2. Find an approximation minimum Steiner tree $T_{A B}$ in $G_{A B}$ spanning the nodes in $V_{D}$.

3. Construct $T$ and $l_{T}$ in $G$ from $T_{A B}$.

End

Let $\rho$ be the approximation ratio of the approximate minimum Steiner tree algorithm used in Algorithm MWMAB.

Lemma 2. The solution delivered by Algorithm MWM-AB for MWM is at most $2 \rho-1$ times the optimum if assumptions $A$ and $B$ hold at the same time.

Proof. Following the definitions of $w_{O P T}^{G}, w_{O P T}^{G_{A B}}, w$, and $w_{A B}$, we have

$$
\begin{aligned}
\frac{w}{w_{O P T}^{G}} \leq & \frac{w_{A B}-(|D|-1)}{w_{O P T}^{G_{A B}}-(|D|-1)} \\
& =\frac{\frac{w_{A B}}{w_{O P T}^{G_{A B}}-\frac{|D|-1}{w_{O P T}^{G_{A B}}}}}{1-\frac{|D|-1}{w_{O P T}^{G_{A B}}}} \\
& \leq \frac{\mid D-\frac{|D|-1}{w_{O P T}^{G_{A B}}}}{1-\frac{|D|-1}{w_{O P T}^{G_{A B}}}}
\end{aligned}
$$




$$
\begin{aligned}
& =\rho+\frac{\rho-1}{\frac{w_{O P T}^{G_{A B}}}{|D|-1}-1} \\
& \leq \rho+\frac{\rho-1}{\frac{2|D|-1}{|D|-1}-1}, \quad \text { since } w_{O P T}^{G_{A B}} \geq|D|-1 \\
& \leq 2 \rho-1 .
\end{aligned}
$$

The best known approximation algorithm for finding a minimum Steiner tree is given by Robins and Zelikovsky [13], which has an approximation ratio of $\rho(\leq 1+\ln 3 / 2)$. We thus have the following corollary.

Corollary 2. Given a network $G$ satisfying assumptions $A$ and $B$, a multicast set $D$, there is an approximation algorithm for the minimum wavelength multicast problem. The solution is at most $(1+\ln 3)$ times of the optimum.

\subsection{Algorithm with Assumption A}

Let the network $G(V, E, \Lambda)$ satisfy assumption $A$ only. This implies that there must be some wavelength covering at least two nodes in $D$ due to the fact that assumption $B$ no longer holds. In the following, we show how to transform $G$ into another network $G^{\prime}$ with a Steiner set $D^{\prime}$ that satisfies assumptions $A$ and $B$. The basic idea is to compress those nodes in $D$ covered by a wavelength into a supernode. This procedure continues until the resulting graph satisfies assumption $B$.

Given $G$, a bipartite graph $H=\left(\Lambda_{H}, V_{H}, E_{H}\right)$ is constructed as follows. $\Lambda_{H}=\left\{v_{\lambda} \mid \lambda \in \Lambda\right\} . V_{H}=\{v \mid v \in$ $V\} . E_{H}=\left\{\left(v_{\lambda}, u\right) \mid\right.$ if $\lambda$ covers $u$ in $\left.G\right\}$. Having the auxiliary graph $H$, select a wavelength $\lambda$ covering the maximum number of nodes in $D$, compress all the nodes that are covered by $\lambda$ into a supernode, add the supernode into $D$ and remove all the covered nodes from $D$ at the same time. Repeat this procedure until no wavelength covers more than one node in $D$ in the resulting graph. The detailed algorithm is described as follows.

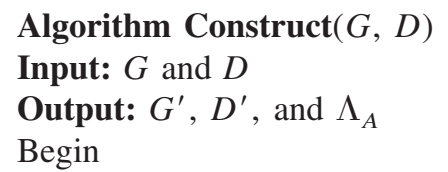

1. $\Lambda_{A} \leftarrow \varnothing$;

2. Construct the bipartite graph $H=\left(\Lambda_{H}, V_{H}, E_{H}\right)$ :

3. while there is $v_{\lambda}$ covering at least two nodes in $D$ do

3.1 Select a wavelength node $v_{\lambda} \in \Lambda_{H}$ covering the maximum number of nodes in $D$;

$3.2 \Lambda_{A} \leftarrow \Lambda_{A} \cup\{\lambda\}$;

$3.3 V_{\lambda} \leftarrow\left\{u \in V_{H} \mid\left(v_{\lambda}, u\right) \in E_{H}\right\}$;

$3.4 V_{H} \leftarrow\left(V_{H} \cup\left\{v_{\lambda}^{\prime}\right\}\right) \backslash V_{\lambda} ; / * v_{\lambda}^{\prime}$ is a supernode */
$3.5 E_{H} \leftarrow E_{H} \cup\left\{\left(u, v_{\lambda}^{\prime}\right) \mid \exists v \in V_{\lambda}, \exists u \in \Lambda_{H} \backslash\left\{v_{\lambda}\right\}\right.$, $\left.(u, v) \in E_{H}\right\}$;

$3.6 E_{H} \leftarrow E_{H} \backslash\left\{(u, v) \in E_{H} \mid v \in V_{\lambda}\right\}$;

$3.7 \Lambda_{H} \leftarrow \Lambda_{H} \backslash\left\{v_{\lambda}\right\}$;

$3.8 D \leftarrow\left(D \cup\left\{v_{\lambda}^{\prime}\right\}\right) \backslash\left(D \cap V_{\lambda}\right)$;

4. Construct $G^{\prime}$ from $G, H$ and $\Lambda_{A}$;

5. $D^{\prime} \leftarrow D$.

End

Recall that $w_{O P T}^{G}$ is the number of wavelengths used in an optimal multicase tree in $G$ under the assumption that the induced subgraph of $G$ by any wavelength is connected. Using a similar argument that is used in the proof of Theorem 2 in [6], the following lemma then follows.

Lemma 3. The size of the wavelength set $\Lambda_{A}$ delivered by Algorithm Construct is at most $(1+2 \log \Delta)$ times of $w_{O P T}^{G}$, where $\Delta$ is the maximum number of nodes in any connected component in the subgraph induced by any single wavelength.

In summary, the algorithm for MWM in a network $G$ satisfying assumption $A$ is presented as follows.

Algorithm MWM-A $(G, D)$

Input: $G=(V, E, \Lambda)$ satisfying assumption $A, D \subseteq V$

Output: $T$ spanning the nodes in $D$ with wavelength assignment function $l_{T}$

Begin

1. Construct $G^{\prime}, D^{\prime}$ and $\Lambda_{A}$ by calling $\operatorname{Construct}(G, D)$.

2. Find a multicast tree $T^{\prime}$ in $G^{\prime}$ covering the nodes in $D^{\prime}$, by calling $\mathrm{MWM}-\mathrm{AB}(G, D)$.

3. Construct a multicast tree $T$ and $l_{T}$ in $G$ covering the nodes in $D$, using $T^{\prime}$ and $\Lambda_{A}$.

End

Let $w_{O P T}^{G^{\prime}}$ be the number of wavelengths in an optimal multicast tree in $G^{\prime}$ and $w^{G^{\prime}}$ the number of wavelengths used in $T^{\prime}$. Let $w$ be the number of wavelengths used in $T$. It is obvious that $w_{O P T}^{G^{\prime}} \leq w_{O P T}^{G}$. By Corollary $2, w^{G^{\prime}} \leq(1$ $+\ln 3) w_{O P T}^{G^{\prime}}$, while $\left|\Lambda_{A}\right| \leq(1+2 \log \Delta) w_{O P T}^{G}$ following Lemma 3. Thus,

$$
\begin{aligned}
w & \leq w^{G^{\prime}}+\left|\Lambda_{A}\right| \\
& \leq(1+\ln 3) w_{O P T}^{G^{\prime}}+(1+2 \log \Delta) w_{O P T}^{G} \\
& \leq(2+\ln 3+2 \log \Delta) w_{O P T}^{G} .
\end{aligned}
$$

Lemma 4. Under the assumption that the subgraph of $G$ induced by any wavelength is connected, there is an approximation algorithm for MWM in $G$, which delivers a solution with an approximation ratio of $(2+\ln 3+2 \log \Delta)$. 


\subsection{Algorithm without Assumptions A and B}

We now provide an approximation algorithm for the case where neither assumption $A$ nor assumption $B$ holds. This implies that there is such a wavelength $\lambda_{i}$ in the network $G$ that the induced subgraph $G_{\lambda_{i}}\left(V_{\lambda_{i}}, E_{\lambda_{i}}\right)$ of $G$ is disconnected. Let $k_{G}\left(\lambda_{i}\right)$ be the number of connected components in $G_{\lambda_{i}}$ and $c_{i, 1}, c_{i, 2}, \ldots, c_{i, k_{G}\left(\lambda_{i}\right)}$ the connected components of $G_{\lambda_{i}}$. The input integer parameter $K$ of the proposed algorithm will be determined later. The algorithm is described as follows.

Algorithm MWM-General $(G, D, K)$

Input: $G=(V, E, \Lambda), D \subseteq V$ and $K$

Output: $T$ spanning the nodes in $D$ with wavelength assignment function $l_{T}$

Begin

1. $G^{\prime} \leftarrow G$ and $D^{\prime} \leftarrow D$.

2. while $\exists \lambda_{i}, k_{G^{\prime}}\left(\lambda_{i}\right)>K$ do

2.1 Compress each of $c_{i, j}$ s as a distinct super-node, $1 \leq j$ $\leq k_{G^{\prime}}\left(\lambda_{i}\right)$. If a connected component contains the nodes in $D^{\prime}$, then add a new super-node into $D^{\prime}$ and remove these nodes in the connected component from $D^{\prime}$ at the same time.

3. $\Lambda_{K} \leftarrow$ the set of compressed wavelengths.

4. for each wavelength $\lambda_{i}$ in $G^{\prime}$ do

4.1 Replace $\lambda_{i}$ in $c_{i, j}$ by a new wavelength $\lambda_{i, j}$ for each $1 \leq j \leq k_{G^{\prime}}\left(\lambda_{i}\right)$.

5. $G^{\prime \prime} \leftarrow$ the resulting graph.

6. $T^{\prime \prime} \leftarrow$ MWM-A $\left(G^{\prime \prime}, D^{\prime}\right)$.

7. Replace the wavelengths in $T^{\prime \prime}$ by their original wavelength, i.e., replace $\lambda_{i, 1}, \lambda_{i, 2}, \ldots, \lambda_{i, k_{G^{\prime}}\left(\lambda_{i}\right)}$ by $\lambda_{i}$. Denote by $T^{\prime}$ the resulting tree.

8. Construct a multicast tree $T_{K}$ in $G$ spanning the nodes in $D$, using $T^{\prime}$ and the wavelengths in $\Lambda_{K}$. End

In Algorithm MWM-General, the number of nodes in the resulting graph will reduced by at least $K$ after each iteration of Step 2. Thus, the number of iterations of Step 2 is no more than $\lceil n / K\rceil$ and $\left|\Lambda_{K}\right| \leq\lceil n / K\rceil$. By Lemma 4, the number of wavelengths in $T^{\prime \prime}$ is no more than $K \cdot(2+\ln$ $3+2 \log \Delta$ ). Therefore, the number of wavelengths in the resulting multicast tree $T_{K}$ is no more than $\lceil n / K\rceil+K \cdot(2$ $+\ln 3+2 \log \Delta) \cdot o p t$, which is minimized when $K$ is equal to $\lceil\sqrt{n /(2+\ln 3+2 \log \Delta) \cdot o p t}\rceil$ approximately. In other words, $\min _{1 \leq K \leq\lfloor n / 2\rfloor}\{\lceil n / K\rceil+K \cdot(2+\ln 3+2$ $\log \Delta) \cdot o p t\}=O(\sqrt{n \cdot \log \Delta \cdot o p t})$.

Theorem 2. There is an approximation algorithm for $M W M$ in a network $G$, which delivers a solution with an approximation ratio of $O(\sqrt{(n \log \Delta) / o p t)}$, where opt is the number of wavelengths used in an optimal solution with 1 $\leq$ opt $\leq n-1$.

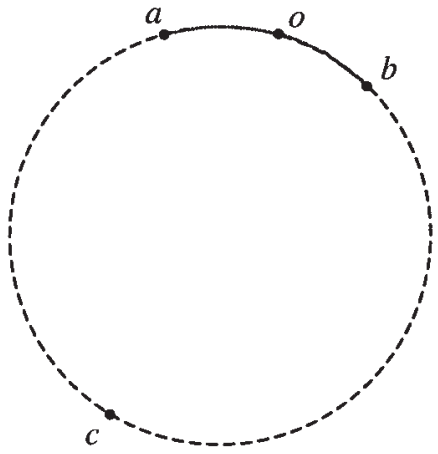

FIG. 2. A counterexample.

\section{CONCLUSIONS}

In this article we have shown that the minimum wavelength multicast problem in a WDM network is not only NP-hard but also hard to approximate. We have also presented an approximation algorithm for it with an approximation ratio of $O(\sqrt{(n \log \Delta) / o p t})$, where $n$ is the number of nodes in the network, $\Delta$ is the maximum number of nodes in a connected component in the subgraph induced by any wavelength, and opt is the number of wavelengths in an optimal solution with $1 \leq$ opt $\leq n-1$.

\section{Acknowledgments}

We appreciate the anonymous referees for their constructive comments and valuable suggestions which have helped improve the quality and presentation of this article.

\section{APPENDIX: A COUNTER-EXAMPLE FOR THEOREM 2 IN [6]}

The basic idea of the approximation algorithm in [6] is to choose a wavelength that can cover the maximum number of nodes in $D$, then replace these covered nodes by a single node in $V$ and $D$, respectively. Repeat this procedure until there is only one node in $D$. The approximation algorithm [6] for MWM in a network satisfying assumption $A$ is shown in the next page.

We show that the approximation ratio of the above algorithm cannot match their claim by a counterexample. As shown in Figure 2, we assume that the network is a ring in which each edge is assigned a distinct wavelength. Consider a multicast set $D$ containing only two nodes $a$ and $b$, shown in Figure 2. The optimal multicast tree is the shortest path between $a$ and $b$, which consists of edges $(a, o)$ and $(o, b)$. Following the algorithm in [6], it may proceed from one of the two choices, that is, it either proceeds along the shortest path and obtains the optimal solution, or it proceeds along the longer path (via node $c$ ) which consists of $n-2$ edges. If it does choose the latter one, the approximate multicast tree obtained by their algorithm requires $n-2$ wavelengths. Thus, the approximation ratio of their algorithm is $\Omega(n)$, not $(1+2 \log \Delta)$ as they claimed. The error 
in their proof is that they assume that there is always a wavelength node $w$ such that $c(w) \leq 1 / 2$, that is, there is a node $w$ covering at least two nodes in $D$ (or $D^{\prime}$ ) during the reduction. In fact, this assumption does not hold in the given example because $c(w)=1$ during the reduction. It seems that this fatal error cannot be fixed. Thus, their algorithm does not deliver the promised solution.

Input A connected graph $G(V, E, w)$ and a multicast request $r(s ; D)$

Output $T$, a routing tree for $r(s ; D)$

Step 0 Initialization $T \leftarrow \varnothing$

Step 1 Construct a bipartite graph $H(V, W ; B)$ set $B \leftarrow\left\{\left(v_{i}, w_{j}\right) \mid\right.$ if $v_{i} \in V\left(w_{j}\right)$, for $i=1, \ldots$, $n, j=1, \ldots, k\}$.

Step 2 Construct $T$ set $D^{\prime} \leftarrow D \cup\{s\}$, while $\left|D^{\prime}\right|>1$ do begin

choose $w \in W$ that can cover the maximum number of vertices in $D^{\prime}$, set $V^{\prime} \leftarrow\{v \in V \mid(v, w) \in B\}$, set $V \leftarrow V V^{\prime} \cup\left\{v^{\prime}\right\}$, set $W \leftarrow W \backslash\{w\}$, set $B \leftarrow B \backslash\{(v, w) \mid v \in V\} \cup\left\{\left(v^{\prime}, w^{\prime}\right) \mid\right.$ if $(v$, $\left.w^{\prime}\right) \in B$, for $v \in V^{\prime}$ and $\left.w^{\prime} \in W \backslash\{w\}\right\}$, set $T \leftarrow T \cup\{e \in E \mid w \in w(e)\}$, set $D^{\prime} \leftarrow D^{\prime} \cup\left\{v^{\prime}\right\} \backslash\left(D^{\prime} \cap V^{\prime}\right)$.

\section{end-while}

Step 3 Modify $T$ into a routing tree for $r(s ; D)$;

Construct a routing tree on $T$ with the minimal number of wavelength conversions,

Return $T$.

\section{REFERENCES}

[1] A. Aggarwal, A. Bar-Noy, D. Coppersmith, R. Ramaswami, B. Schieber, and M. Sudan, Efficient routing in optical networks. J ACM 46 (1996), 973-1001.

[2] B. Chen and J. Wang, Efficient routing and wavelength assignment for multicast in WDM networks, IEEE J Selected Areas Commun 20 (2002), 97-109.
[3] I. Chlamtac, A. Farago, and T. Zhang, Lightpath (wavelength) routing in large WDM networks. IEEE J Selected Areas Commun 14 (1996), 909-913.

[4] Y. Dodis and S. Khanna, Designing networks with bounded pairwise distance, Proc ACM STOC'99, Atlanta, GA, May 1999, pp. 750-759.

[5] X. Jia, D. Du, and X. Hu, Integrated algorithms for delay bounded multicast routing and wavelength assignment in all optical networks, Comput Commun 24 (2001), 1390-1399.

[6] D. Li, X. Du, X. Hu, L. Ruan, and X. Jia, Minimizing number of wavelengths in multicast routing trees in WDM networks, Networks 35 (2000), 260-265.

[7] W. Liang and X. Shen, Improved lightpath (wavelength) routing in large WDM networks, IEEE Trans Commun 48 (2000), 1571-1579.

[8] W. Liang and H. Shen, Multicasting and broadcasting in large WDM networks, Proc of 12th Int'l Conf on Parallel Processing Symp, IEEE Computer Society Press, Los Alamitos, CA, 1998, pp. 365-369.

[9] R. Liberskind-Hadas and R. Melhem, Multicast routing and wavelength assignment in multi-hop optical networks, IEEE/ACM Trans Networking 10 (2002), 621-629.

[10] B. Mukherjee, WDM-based local lightwave networks, Part I: Single-hop systems, IEEE Network 6 (1992), 12-27.

[11] B. Mukherjee, WDM-based local lightwave networks, Part II: Multi-hop systems, IEEE Network 6 (1992), 20-32.

[12] R. Ramaswami, Multi-wavelength lightwave networks for computer communication, IEEE Commun Mag 31 (1993), $78-88$.

[13] G. Robins and A. Zelikovsky, Improved Steiner tree approximation in graphs, Proc of 11th Annual ACM-SIAM Symp on Discrete Algorithms, ACM Press, New York, NY, 2000, pp. 770-779.

[14] R.J. Vitter and D.H.C. Du, Distributed computing with high-speed optical networks, IEEE Comput 26 (1993), $8-18$.

[15] X. Zhang, J. Wei, and C. Qiao, Constrained multicast routing in WDM networks with sparse light splitting, Proc of IEEE INFOCOM'00, 2000, pp. 1781-1790.

[16] T.F. Znati, T. Alrabiah, and R. Melhem, Low-cost delaybounded point-to-point multipoint communication to support multicasting over WDM networks, Comput Networks 38 (2002), 423-445. 